\title{
Tensor Forces and the Ground-State Structure of Nuclei
}

\author{
R. Schiavilla ${ }^{1,2}$, R.B. Wiringa ${ }^{3}$, Steven C. Pieper ${ }^{3}$, and J. Carlson ${ }^{4}$ \\ ${ }^{1}$ Jefferson Lab, Newport News, VA 23606 \\ ${ }^{2}$ Department of Physics, Old Dominion University, Norfolk, VA 23529 \\ ${ }^{3}$ Physics Division, Argonne National Laboratory, Argonne, IL 61801 \\ ${ }^{4}$ Theoretical Division, Los Alamos National Laboratory, Los Alamos, NM 87545
}

(Dated: August 17, 2018)

\begin{abstract}
Two-nucleon momentum distributions are calculated for the ground states of nuclei with mass number $A \leq 8$, using variational Monte Carlo wave functions derived from a realistic Hamiltonian with two- and three-nucleon potentials. The momentum distribution of $n p$ pairs is found to be much larger than that of $p p$ pairs for values of the relative momentum in the range (300-600) $\mathrm{MeV} / \mathrm{c}$ and vanishing total momentum. This order of magnitude difference is seen in all nuclei considered and has a universal character originating from the tensor components present in any realistic nucleonnucleon potential. The correlations induced by the tensor force strongly influence the structure of $n p$ pairs, which are predominantly in deuteron-like states, while they are ineffective for $p p$ pairs, which are mostly in ${ }^{1} \mathrm{~S}_{0}$ states. These features should be easily observable in two-nucleon knock-out processes, such as $A\left(e, e^{\prime} n p\right)$ and $A\left(e, e^{\prime} p p\right)$.
\end{abstract}

PACS numbers: 21.60.-n,21.30.Fe,25.30.-c

The two preeminent features of the nucleon-nucleon $(N N)$ interaction are its short-range repulsion and intermediate- to long-range tensor character. These induce strong spatial-spin-isospin $N N$ correlations, which leave their imprint on the structure of ground- and excited-state wave functions. Several nuclear properties reflect the presence of these features. For example, the two-nucleon density distributions $\rho_{T S}^{M_{S}}(\mathbf{r})$ in states with pair spin $S=1$ and isospin $T=0$ are very small at small inter-nucleon separation $\mathbf{r}$ and exhibit strong anisotropies depending on the spin projection $M_{S}$ [1]. Nucleon momentum distributions $N(k)$ [2, 3] and spectral functions $S(k, E)$ [4] have large high-momentum and, in the case of $S(k, E)$, high-energy components, which are produced by short-range and tensor correlations. The latter also influence the distribution of strength in response functions $R(k, \omega)$, which characterize the response of the nucleus to a spin-isospin disturbance injecting momentum $\mathbf{k}$ and energy $\omega$ into the system [5, 6]. Lastly, calculations of low-energy spectra in light nuclei (up to mass number $A=10$ ) have demonstrated that tensor forces play a crucial role in reproducing the observed ordering of the levels and, in particular, the observed absence of stable $A=8$ nuclei [7].

In the present study we show that tensor correlations also impact strongly the momentum distributions of $N N$ pairs in the ground state of a nucleus and, in particular, that they lead to large differences in the $n p$ versus $p p$ distributions at moderate values of the relative momentum in the pair. These differences should be observable in two-nucleon knock-out processes, such as $A\left(e, e^{\prime} n p\right)$ and $A\left(e, e^{\prime} p p\right)$ reactions.

The probability of finding two nucleons with relative momentum $\mathbf{q}$ and total momentum $\mathbf{Q}$ in isospin state $T M_{T}$ in the ground state of a nucleus is proportional to the density

$$
\begin{aligned}
\rho_{T M_{T}}(\mathbf{q}, \mathbf{Q})= & \frac{A(A-1)}{2(2 J+1)} \sum_{M_{J}} \int d \mathbf{r}_{1} d \mathbf{r}_{2} d \mathbf{r}_{3} \cdots d \mathbf{r}_{A} d \mathbf{r}_{1}^{\prime} d \mathbf{r}_{2}^{\prime} \psi_{J M_{J}}^{\dagger}\left(\mathbf{r}_{1}^{\prime}, \mathbf{r}_{2}^{\prime}, \mathbf{r}_{3}, \ldots, \mathbf{r}_{A}\right) \\
& \times e^{-i \mathbf{q} \cdot\left(\mathbf{r}_{12}-\mathbf{r}_{12}^{\prime}\right)} e^{-i \mathbf{Q} \cdot\left(\mathbf{R}_{12}-\mathbf{R}_{12}^{\prime}\right)} P_{T M_{T}}(12) \psi_{J M_{J}}\left(\mathbf{r}_{1}, \mathbf{r}_{2}, \mathbf{r}_{3}, \ldots, \mathbf{r}_{A}\right),
\end{aligned}
$$

where $\mathbf{r}_{12} \equiv \mathbf{r}_{1}-\mathbf{r}_{2}, \mathbf{R}_{12} \equiv\left(\mathbf{r}_{1}+\mathbf{r}_{2}\right) / 2$, and similarly for $\mathbf{r}_{12}^{\prime}$ and $\mathbf{R}_{12}^{\prime}$. $P_{T M_{T}}(12)$ is the isospin projection operator, and $\psi_{J M_{J}}$ denotes the nuclear wave function in spin and spin-projection state $J M_{J}$. The normalization is

$$
\int \frac{d \mathbf{q}}{(2 \pi)^{3}} \frac{d \mathbf{Q}}{(2 \pi)^{3}} \rho_{T M_{T}}(\mathbf{q}, \mathbf{Q})=N_{T M_{T}},
$$

where $N_{T M_{T}}$ is the number of $N N$ pairs in state $T M_{T}$. Obviously, integrating $\rho_{T M_{T}}(\mathbf{q}, \mathbf{Q})$ over only $\mathbf{Q}$ gives the probability of finding two nucleons with relative momentum q, regardless of their pair momentum $\mathbf{Q}$ (and viceversa).

The present study of two-nucleon momentum distributions in light nuclei (up to $A=8$ ) is based on variational Monte Carlo (VMC) wave functions, derived from a realistic Hamiltonian consisting of the Argonne $v_{18}$ twonucleon [8] and Urbana-IX three-nucleon [9] interactions (AV18/UIX). The high accuracy of the VMC wave func- 
tions is well documented (see Refs. [10, 11] and references therein), as is the quality of the AV18/UIX Hamiltonian in quantitatively accounting for a wide variety of light nuclei properties, such as elastic and inelastic electromagnetic form factors [12], and low-energy capture reactions [13]. However, it is important to stress that the large effect of tensor correlations on two-nucleon momentum distributions and the resulting isospin dependence of the latter remain valid, even if one uses a semi-realistic Hamiltonian model. This will be shown explicitly below.
The double Fourier transform in Eq. (11) is computed by Monte Carlo (MC) integration. A standard Metropolis walk, guided by $\left|\psi_{J M_{I}}\left(\mathbf{r}_{1}, \mathbf{r}_{2}, \mathbf{r}_{3}, \ldots, \mathbf{r}_{A}\right)\right|^{2}$, is used to sample configurations [11]. For each configuration a twodimensional grid of Gauss-Legendre points, $x_{i}$ and $X_{j}$, is used to compute the Fourier transform. Instead of just moving the $\psi^{\prime}$ position $\left(\mathbf{r}_{12}^{\prime}\right.$ and $\left.\mathbf{R}_{12}^{\prime}\right)$ away from a fixed $\psi$ position ( $\mathbf{r}_{12}$ and $\mathbf{R}_{12}$ ), both positions are moved symmetrically away from $\mathbf{r}_{12}$ and $\mathbf{R}_{12}$, so Eq. (1) becomes

$$
\begin{aligned}
\rho_{T M_{T}}(\mathbf{q}, \mathbf{Q})=\frac{A(A-1)}{2(2 J+1)} \sum_{M_{J}} \int & d \mathbf{r}_{1} d \mathbf{r}_{2} d \mathbf{r}_{3} \cdots d \mathbf{r}_{A} d \mathbf{x} d \mathbf{X} \psi_{J M_{J}}^{\dagger}\left(\mathbf{r}_{12}+\mathbf{x} / 2, \mathbf{R}_{12}+\mathbf{X} / 2, \mathbf{r}_{3}, \ldots, \mathbf{r}_{A}\right) \\
& \times e^{-i \mathbf{q} \cdot \mathbf{x}} e^{-i \mathbf{Q} \cdot \mathbf{X}} P_{T M_{T}}(12) \psi_{J M_{J}}\left(\mathbf{r}_{12}-\mathbf{x} / 2, \mathbf{R}_{12}-\mathbf{X} / 2, \mathbf{r}_{3}, \ldots, \mathbf{r}_{A}\right)
\end{aligned}
$$

Here the polar angles of the $x$ and $X$ grids are also sampled by MC integration, with one sample per pair. This procedure is similar to that adopted most recently in studies of the ${ }^{3} \mathrm{He}\left(e, e^{\prime} p\right) d$ and ${ }^{4} \mathrm{He}\left(\vec{e}, e^{\prime} \vec{p}\right)^{3} \mathrm{H}$ reactions [14], and has the advantage of very substantially reducing the statistical errors originating from the rapidly oscillating nature of the integrand for large values of $q$ and $Q$. Indeed, earlier calculations of nucleon and cluster momentum distributions in few-nucleon systems, which were carried out by direct MC integration over all coordinates, were very noisy for momenta beyond $2 \mathrm{fm}^{-1}$, even when the random walk consisted of a very large number of configurations [2].

The present method is, however, computationally intensive, because complete Gaussian integrations have to be performed for each of the configurations sampled in the random walk. The large range of values of $x$ and $X$ required to obtain converged results, especially for ${ }^{3} \mathrm{He}$, require fairly large numbers of points; we used grids of up to 96 and 80 points for $x$ and $X$, respectively. We also sum over all pairs instead of just pair 12 .

The $n p$ and $p p$ momentum distributions in ${ }^{3} \mathrm{He},{ }^{4} \mathrm{He}$, ${ }^{6} \mathrm{Li}$, and ${ }^{8} \mathrm{Be}$ nuclei are shown in Fig. 1 as functions of the relative momentum $q$ at fixed total pair momentum $Q=0$, corresponding to nucleons moving back to back. The statistical errors due to the Monte Carlo integration are displayed only for the $p p$ pairs; they are negligibly small for the $n p$ pairs. The striking features seen in all cases are: i) the momentum distribution of $n p$ pairs is much larger than that of $p p$ pairs for relative momenta in the range $1.5-3.0 \mathrm{fm}^{-1}$, and ii) for the helium and lithium isotopes the node in the $p p$ momentum distribution is absent in the $n p$ one, which instead exhibits a change of slope at a characteristic value of $p \simeq 1.5 \mathrm{fm}^{-1}$. The nodal structure is much less prominent in ${ }^{8} \mathrm{Be}$. At small values of $q$ the ratios of $n p$ to $p p$ momentum distributions are closer to those of $n p$ to $p p$ pair numbers, which in
${ }^{3} \mathrm{He},{ }^{4} \mathrm{He},{ }^{6} \mathrm{Li}$, and ${ }^{8} \mathrm{Be}$ are respectively $2,4,3$, and $8 / 3$. Note that the $n p$ momentum distribution is given by the linear combination $\rho_{T M_{T}=10}+\rho_{T M_{T}=00}$, while the $p p$ momentum distribution corresponds to $\rho_{T M_{T}=11}$. The wave functions utilized in the present study are eigenstates of total isospin $\left(1 / 2\right.$ for ${ }^{3} \mathrm{He}$, and 0 for ${ }^{4} \mathrm{He},{ }^{6} \mathrm{Li}$, and $\left.{ }^{8} \mathrm{Be}\right)$, so the small effects of isospin-symmetry-breaking interactions are ignored. As a result, in ${ }^{4} \mathrm{He},{ }^{6} \mathrm{Li}$, and ${ }^{8} \mathrm{Be}$ the $\rho_{T M_{T}}$ is independent of the isospin projection and, in particular, the $p p$ and $T=1 n p$ momentum distributions are the same.

The excess strength in the $n p$ momentum distribution is due to the strong correlations induced by tensor components in the underlying $N N$ potential. For $Q=0$, the pair and residual $(A-2)$ system are in a relative $\mathrm{S}$-wave. In ${ }^{3} \mathrm{He}$ and ${ }^{4} \mathrm{He}$ with uncorrelated wave functions, $3 / 4$ of the $n p$ pairs are in deuteron-like $T, S=0,1$ states, while

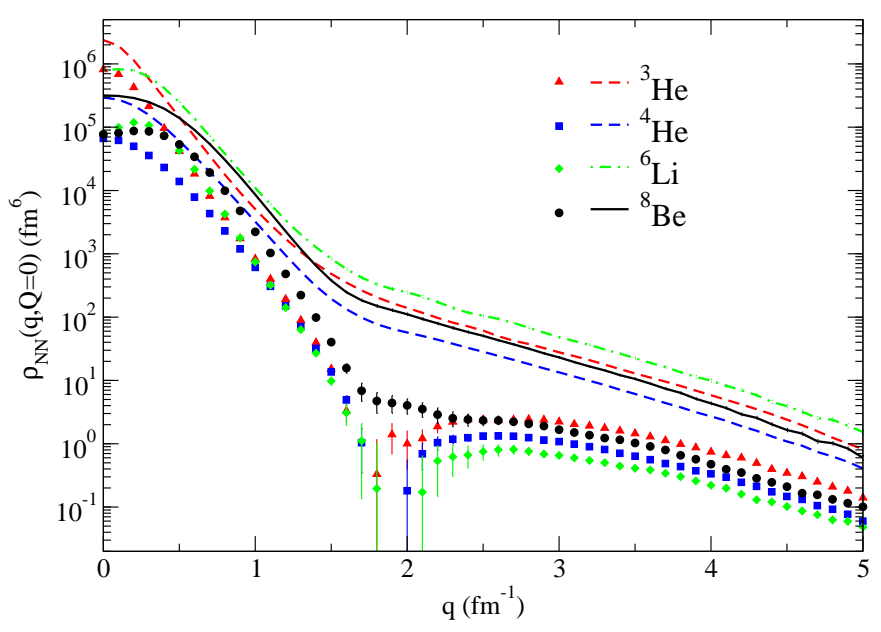

FIG. 1: (Color online) The $n p$ (lines) and $p p$ (symbols) momentum distributions in various nuclei as functions of the relative momentum $q$ at vanishing total pair momentum $Q$. 
the $p p, n n$ and remaining $1 / 4$ of $n p$ pairs are in $T, S=1,0$ (quasi-bound) states. When multi-body tensor correlations are taken into account, $10-15 \%$ of the $T, S=1,0$ pairs are spin-flipped to $T, S=1,1$ pairs, but the number of $T, S=0,1$ pairs hardly changes [1]. In $A>4 \mathrm{nu}-$ clei, some $n p$ and $p p$ pairs will be in relative $\mathrm{P}$-waves ( $T, S=1,1$ and 0,0$)$ when one particle is in the s-shell and one in the p-shell. Nevertheless, 5.5 out of $9 \mathrm{np}$ pairs in ${ }^{6} \mathrm{Li}\left(9\right.$ out of 16 in ${ }^{8} \mathrm{Be}$ ) are expected to be deuteron-like, while half the $p p$ pairs will be in $T, S=1,0$ states and half in $T, S=1,1$ states [15]. The tensor force vanishes in the former and is weak in the latter.

These expectations are born out by our calculations, as Fig. 1 clearly demonstrates. The $n p$ momentum distributions for $q$ values larger than $1.5 \mathrm{fm}^{-1}$ only differ by a scaling factor, and indeed all scale relative to the deuteron momentum distribution as shown in Fig. 2, The deuteron $\rho_{n p}(q, Q=0)$ has been scaled to match that of ${ }^{4} \mathrm{He}$ at $q=1.5 \mathrm{fm}^{-1}$. (The scaling property does not extend to the low $q$ region, because there binding effects take over.) The S- and D-wave components of the deuteron density are also shown in the figure as dotted lines; the D-state component is the dominant part over the range $1.4-4.0 \mathrm{fm}^{-1}$, while the S-state component has a node at $2.1 \mathrm{fm}^{-1}$.

Similar considerations in relation to scaling and binding effects also remain valid when considering the $p p$ momentum distributions. In particular, the node seen in helium and lithium is reminiscent of the node in the Swave momentum distribution (shown in Fig. 21). In larger nuclei the node is filled in, due to the fact that $p p$ pairs are not exclusively in quasi-bound states, but can also be in $\mathrm{P}$-wave or higher partial-wave states.

Figure 2 also shows the $n p$ and $p p$ momentum distri-

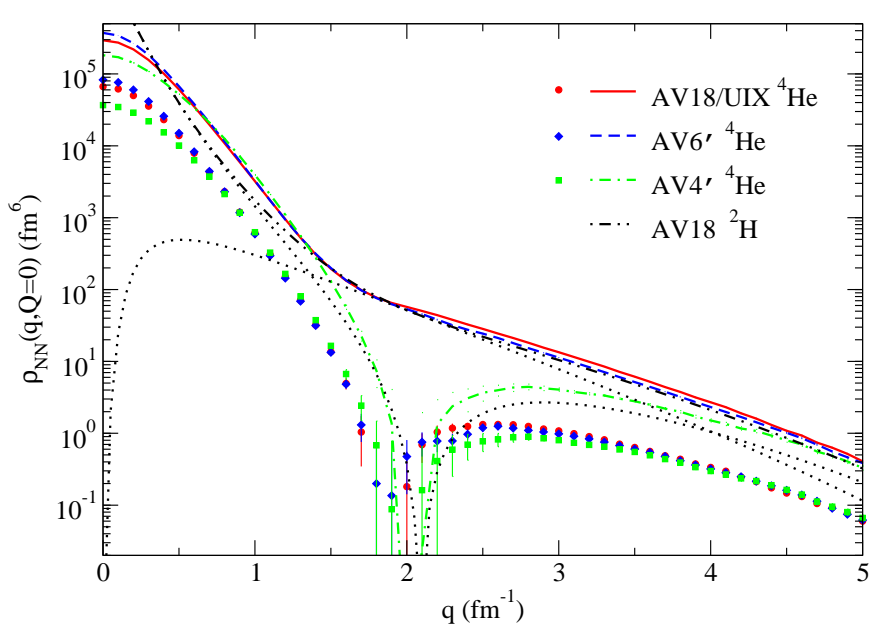

FIG. 2: (Color online) The $n p$ (lines) and $p p$ (symbols) momentum distributions in ${ }^{4} \mathrm{He}$ obtained with different Hamiltonians. Also shown is the scaled momentum distribution for the AV18 deuteron; its separate S- and D-wave components are shown by dotted lines. butions in ${ }^{4} \mathrm{He}$ obtained with Hamiltonians of decreasing sophistication, ranging from the fully realistic AV18/UIX model to the semi-realistic Argonne $v_{6}^{\prime}\left(\mathrm{AV}^{\prime}\right)$ to the relatively simple Argonne $v_{4}^{\prime}\left(\mathrm{AV} 4^{\prime}\right)$. The $\mathrm{AV} 4^{\prime}$ and $\mathrm{AV} 6^{\prime}$ potentials are constructed to preserve as many features of $N N$ scattering and deuteron properties as feasible [7]. The AV4' has only central, spin, and isospin operators with no tensor component: it reproduces the ${ }^{1} \mathrm{~S}_{0}$ phase shift, and the deuteron binding energy but with only an S-state component; the D-state, induced by the tensor term in the potential and associated with one-pion exchange at long range, is absent. On the other hand, the $\mathrm{AV} 6^{\prime}$ includes tensor terms and produces a bound deuteron quite close to that of AV18. These features of the underlying $N N$ potential, or the lack of them, are reflected in the calculated momentum distributions. In particular, note the node which develops in the $\mathrm{AV} 4^{\prime} n p$ momentum distribution, due to the purely S-wave nature of the deuteron-like state. On the other hand, the $\mathrm{AV} 6^{\prime}$ and AV18/UIX results are very close to each other, demonstrating the essential role played by the tensor potential in substantially increasing the intermediate-momentum components of $n p$ pairs.

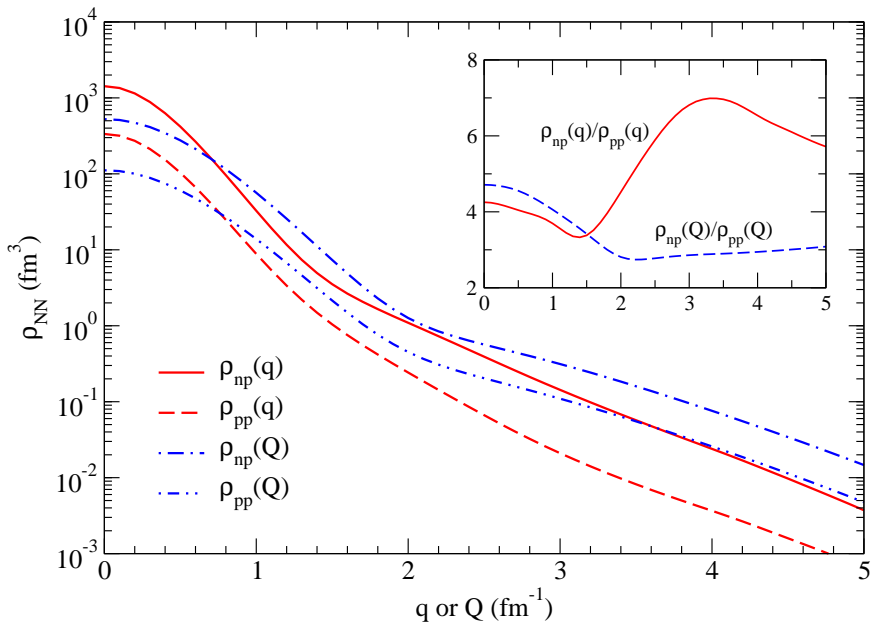

FIG. 3: (Color online) The momentum distributions $\rho_{N N}(q)$ and $\rho_{N N}(Q)$ in ${ }^{4} \mathrm{He}$ for $n p$ and $p p$. The inset shows the ratios $\rho_{n p}(q) / \rho_{p p}(q)$ and $\rho_{n p}(Q) / \rho_{p p}(Q)$.

The momentum distributions $\rho_{N N}(q)$ and $\rho_{N N}(Q)$ obtained by integrating over $Q$ or $q$ respectively are plotted in Fig. 3 for ${ }^{4} \mathrm{He}$ with the AV18/UIX Hamiltonian. There is no node in $\rho_{p p}(q)$ : it is filled in by the contributions of $p p$ channels other than ${ }^{1} \mathrm{~S}_{0}$. These channels are now allowed, because the orbital angular momentum between the $p p$ pair and the residual $n n$ cluster is not constrained to vanish for $Q>0$. A remnant of the strong tensor correlations affecting $\rho_{n p}(q)$ still persists. This is illustrated in the inset of Fig. 3, which shows the ratio $\rho_{n p}(q) / \rho_{p p}(q)$. However, the effect is far less dramatic than in the back-to-back $(Q=0)$ kinematics. 
Note that $\rho_{N N}(q)$ and $\rho_{N N}(Q)$ have the same normalization, i.e. $(A-Z) Z$ for $n p$ and $Z(Z-1) / 2$ for $p p$. The $\rho_{n p}(Q) / \rho_{p p}(Q)$ ratio is close to the $n p$ to $p p$ pair number ratio -4 in ${ }^{4} \mathrm{He}$ - over the whole range of $Q$ values. This holds true for all the nuclei studied.

The most direct evidence for tensor correlations in nuclei comes from measurements of the deuteron structure functions and tensor polarization by elastic electron scattering [16]. In essence, these measurements have mapped out the Fourier transforms of the charge densities of the deuteron in states with spin projections \pm 1 and 0 , showing that they are very different. In other processes, such as ${ }^{2} \mathrm{H}(d, \gamma)^{4} \mathrm{He}$ [17] at very low energy, or proton knockout from a polarized deuteron [18] (as well as in the nuclear properties mentioned at the beginning of this letter), the effects of tensor correlations are more subtle and their presence is not easily isolated in the experimental data. This is because of "contaminations" from initial or final state interactions and many-body terms in the transition operators.

Some of these corrections will also pollute the cross sections for $\left(e, e^{\prime} n p\right)$ and $\left(e, e^{\prime} p p\right)$, or $(p, p p)$ and $(p, p p n)$, knock-out processes in back-to-back kinematics. However, one would expect the contributions due to final state interactions in the $n p$ and $p p$ reactions, both between the nucleons in the pair and between these and the nucleons in the residual $(A-2)$ system, to be of similar magnitude for relative momenta in the range (300-600) $\mathrm{MeV} / \mathrm{c}$. In the electrodisintegration processes, the leading electromagnetic two-body currents associated with pion and $\rho$-meson exchange, denoted respectively as $P S$ and $V$ in Ref. [19], vanish in $p p$ because of their isospin structure. Of course, they will contribute in $n p$, but are not expected to produce large effects. Thus the ratio of $n p$ to $p p$ cross sections should be much larger than unity for relative momenta within (300-600) MeV/c, reflecting the large difference between the corresponding momentum distributions in this range. There are strong indications from a recent analysis of a BNL experiment, which measured cross sections for $(p, p p)$ and $(p, p p n)$ processes on ${ }^{12} \mathrm{C}$ in kinematics close to two nucleons being ejected back to back, that this is indeed the case 20]. The ratio of $(p, p p n)$ to $(p, p p)$ events over the range of relative momenta $(275-550) \mathrm{MeV} / \mathrm{c}$ is found to be roughly 20, albeit with a rather large error. Hopefully, a more precise value for this ratio will become available in the near future, when the analysis of ${ }^{12} \mathrm{C}\left(e, e^{\prime} n p\right)$ and ${ }^{12} \mathrm{C}\left(e, e^{\prime} p p\right)$ data, taken at Jefferson Lab, is completed [21].

It would be interesting to extend these measurements to other nuclei. In ${ }^{3} \mathrm{He}$ and ${ }^{4} \mathrm{He}$, one would expect the node in the $p p$ momentum distribution to be filled in by interaction effects in the final state [14]. However, the ratio of $n p$ to $p p$ cross sections in the range (300$600) \mathrm{MeV} / \mathrm{c}$ should still reflect the large value of the $n p$ momentum distribution at these values of relative momenta. This would provide a further, direct verification of the crucial role that the tensor force plays in shaping the short-range structure of nuclei.

We dedicate this paper to the memory of Vijay R. Pandharipande, a mentor and friend, who was deeply interested in evidence for correlations in nuclei. This is also in remembrance of our colleague and friend Adelchi Fabrocini, who contributed significantly to the theoretical study of correlations in strongly interacting systems. An interesting and stimulating conversation with D. Higinbotham and E. Piasetzky is gratefully acknowledged by one of the authors (R.S.). This work is supported by the U.S. Department of Energy, Office of Nuclear Physics, under contracts DE-AC05-06OR23177 (R.S.), DE-AC02-06CH11357 (S.C.P. and R.B.W.), and W-7405-ENG36 (J.C.). The calculations were made at Argonne's Laboratory Computing Resource Center.

[1] J. L. Forest et al., Phys. Rev. C 54, 646 (1996).

[2] R. Schiavilla, V. R. Pandharipande, and R. B. Wiringa, Nucl. Phys. A 449, 219 (1986).

[3] S. C. Pieper, R. B. Wiringa, and V. R. Pandharipande, Phys. Rev. C 46, 1741 (1992).

[4] O. Benhar, A. Fabrocini, and S. Fantoni, Nucl. Phys. A 505, 267 (1989).

[5] A. Fabrocini and S. Fantoni, Nucl. Phys. A 503, 375 (1989).

[6] V. R. Pandharipande et al., Phys. Rev. C 49, 789 (1994).

[7] R. B. Wiringa and S. C. Pieper, Phys. Rev. Lett. 89, 182501 (2002).

[8] R. B. Wiringa, V. G. J. Stoks, and R. Schiavilla, Phys. Rev. C 51, 38 (1995).

[9] B. S. Pudliner et al., Phys. Rev. Lett. 74, 4396 (1995).

[10] J. Carlson and R. Schiavilla, Rev. Mod. Phys. 70, 743 (1998).

[11] S. C. Pieper and R. B. Wiringa, Annu. Rev. Nucl. Part. Sci. 51, 53 (2001).

[12] R. B. Wiringa and R. Schiavilla, Phys. Rev. Lett. 81, 4317 (1998); L. E. Marcucci, D. O. Riska, and R. Schiavilla, Phys. Rev. C 58, 3069 (1998).

[13] L. E. Marcucci et al., Nucl. Phys. A 777, 111 (2006).

[14] R. Schiavilla et al., Phys. Rev. Lett. 94, 072303 (2005); Phys. Rev. C 72, 064003 (2005).

[15] R. B. Wiringa, Phys. Rev. C 73, 034317 (2006).

[16] A complete list of references is in R. Gilman and F. Gross, J. Phys. G28, R37 (2002).

[17] A. Arriaga, V. R. Pandharipande, and R. Schiavilla, Phys. Rev. C 43, 983 (1991).

[18] Z.-L. Zhou et al., Phys. Rev. Lett. 82, 687 (1999); I. Passchier et al., Phys. Rev. Lett. 88, 102302 (2002).

[19] L. E. Marcucci et al., Phys. Rev. C 72, 014001 (2005).

[20] E. Piasetzky et al., Phys. Rev. Lett. 97, 162504 (2006).

[21] E. Piasetzky and D. Higinbotham, private communication. 